\title{
AMOSTRAGEM DE COMPOSTOS ORGÂNICOS VOLÁTEIS NO AR UTILIZANDO A TÉCNICA DE MICROEXTRAÇÃO EM FASE SÓLIDA
}

\author{
Fabrício Vilela Parreira" e Zenilda de Lourdes Cardeal* \\ Departamento de Química, Instituto de Ciências Exatas, Universidade Federal de Minas Gerais, CP 702, \\ 31270-901 Belo Horizonte - MG
}

Recebido em 4/5/04; aceito em 4/11/04; publicado na web em 28/2/05

\begin{abstract}
Sampling of volatile organic compounds in air by solid phase microextraction. The sampling of volatile organic compounds using solid phase microextraction is reviewed and its principles are described. The development and application of solid phase microextraction in the sampling of VOCs are presented and discussed.
\end{abstract}

Keywords: SPME sampling; VOC; fundamental principles and applications.

Os compostos orgânicos voláteis (COV) têm várias definições apresentadas por diferentes autores. De acordo com Keith ${ }^{1}$, COV são compostos orgânicos que apresentam como característica um valor de pressão de vapor maior que $0,01 \mathrm{kPa}$. Além da pressão de vapor, o ponto de ebulição também tem sido uma outra propriedade física utilizada para classificar compostos orgânicos presentes na atmosfera. Isto é, COV são compostos que apresentam valores de ponto de ebulição abaixo $150^{\circ} \mathrm{C}$. Os COV tem uma sub-categoria, são os chamados compostos orgânicos muito voláteis (COMV), que possuem pressão de vapor maior que $15 \mathrm{kPa}^{1}$.

A amostragem de COV no ar depende das propriedades químicas e físicas dos compostos, da concentração presente no ar, da umidade relativa e da temperatura, entre outros. Desta forma, existem diferentes procedimentos para amostragem de COV, que podem ser classificados de modo geral em métodos com e sem préconcentração do analito. No método sem pré-concentração, o ar é coletado em recipientes, sem nenhum procedimento de concentração durante a coleta. Os recipientes mais usados são confeccionados em polímero, vidro ou aço inoxidável. A principal desvantagem deste método é que só pode ser aplicado quando as concentrações de COV são elevadas. Quando componentes traços precisam ser analisados, o ar contido nestes recipientes deve ser submetido a um processo de concentração para aumentar a sensibilidade. A amostragem de COV através do procedimento de pré-concentração pode ser realizada por sistemas de criogênia e, também, por utilização de fases sólidas ou líquidas. A amostragem por fases sólidas é um dos procedimentos mais utilizados para COV no $\mathrm{ar}^{1}$. Os analitos são extraídos e concentrados através da passagem de ar sobre uma fase sólida, tal como carvão ativado, carbono grafitizado ou polímero poroso e, em seguida, os compostos são dessorvidos por temperatura ou solvente. As técnicas atuais de pré-concentração requerem equipamentos de custo elevado, utilizam solventes tóxicos e consomem muito tempo ${ }^{2,3}$.

A técnica de microextração em fase sólida ("SPME-solid phase microextraction") é um método alternativo, extremamente atraente e fácil de operar. Ela combina amostragem e pré-concentração dos analitos num único processo, além de possibilitar a dessorção

*e-mail: zenilda@ufmg.br

\# Endereço permanente: Setor de Medições Ambientais, Fundação Centro Tecnológico de Minas Gerais, Belo Horizonte - MG. direta no sistema cromatográfico. As vantagens da SPME em relação aos métodos tradicionais de amostragem de ar são baixo custo de análise, reutilização da fibra, sistema portátil, facilidade de operação, facilidade para automação, perda e contaminação mínima da amostra durante o transporte e armazenamento e, finalmente, existência de grande diversidade de fases para serem aplicadas em diferentes classes de compostos.

A técnica de extração foi desenvolvida por Arthur e Pawliszyn em $1990^{4}$. Neste primeiro trabalho, o dispositivo de SPME era baseado numa microseringa Hamilton série 7000 (Figura 1). De 1990 até os dias atuais, o sistema de SPME foi aperfeiçoado e, atualmente, consiste de uma haste de sílica fundida de $10 \mathrm{~mm}$ de comprimento recoberta por $300 \mu \mathrm{m}$ de espessura de fase sólida ou recoberta com um filme fino de um líquido polimérico, que apresenta diferentes espessuras.

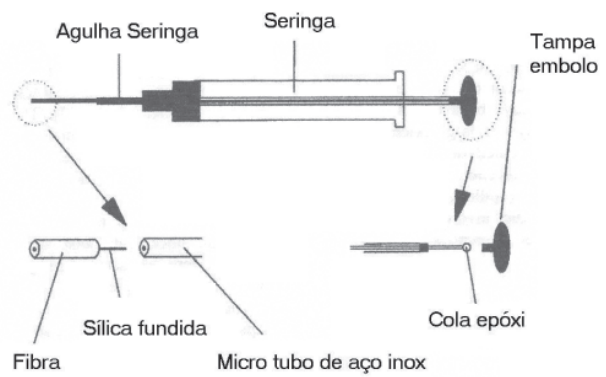

Figura 1. Primeiro dispositivo de SPME construído com seringas Hamilton. Adaptado da ref. 15

A amostragem é baseada no equilíbrio de absorção e/ou adsorção dos analitos entre uma micro fase de coleta, que pode ser sólida ou líquida, e a matriz contendo os analitos.

Esta técnica pode ser aplicada na amostragem de substratos ambientais, tais como águas, efluentes industriais, sólidos e ga$\operatorname{ses}^{5-7}$. A SPME tem sido muito utilizada para análise de amostras líquidas, enquanto que para amostras gasosas há poucos trabalhos publicados, como observado por Fox ${ }^{8}$.

Quando aplicada à amostragem de compostos no ar, a fibra de SPME pode ser utilizada recolhida dentro da agulha da seringa ou exposta diretamente ao ar. Em ambos os métodos, a técnica de SPME pode ser usada nos modos de amostragem ativa e passiva. No caso de amostragem com a fibra recolhida dentro da agulha da 
seringa de SPME e coleta no modo passivo, a técnica comporta-se como um amostrador de longo período9. O tempo de equilíbrio pode levar dias, o que torna a técnica SPME aplicável ao monitoramento de longo período ou em estudo de saúde ocupacional. Além deste tipo de monitoramento, a técnica SPME é também aplicada para amostragem em situações de não equilíbrio ${ }^{10}$ e em amostragem rápida ${ }^{11}$.

A análise dos compostos presentes na fibra de SPME consiste na introdução do dispositivo de SPME no injetor de um CG para dessorção térmica ou inserção em uma válvula de injeção de um cromatógrafo em fase líquida, onde os analitos são dessorvidos por solventes e introduzidos no cromatógrafo para separação e detecção. Após a dessorção no injetor, a fibra pode ser reutilizada para outra amostragem, desde que seja condicionada previamente.

Se o ensaio não for realizado logo após a coleta, alguns cuidados devem ser tomados, como proteção física da fibra e preservação adequada da amostra coletada. É importante que a fibra tenha proteção antes, durante e após a amostragem, evitando que seja destruída, devido a sua estrutura frágil. A preservação da amostra coletada na fibra é fundamental pois o tempo e a temperatura de armazenamento podem ser cruciais para a qualidade dos resultados, especialmente na coleta de COV. As duas formas de prevenir a perda de COV após amostragem são a utilização de septo na ponta da agulha ${ }^{12} \mathrm{e}$, ao mesmo tempo, resfriamento da fibra ${ }^{13}$.

\section{DISPOSITIVOS E FIBRAS DO SISTEMA SPME}

Atualmente, existem três tipos de dispositivos de SPME disponíveis no mercado, sendo um destinado à injeção manual, outro para amostragem em campo e o último, para injeção automática no CG ou para análise por cromatografia líquida. O dispositivo para injeção manual pode ser utilizado para amostragem em campo, desde que certos cuidados sejam observados (Figura 2).

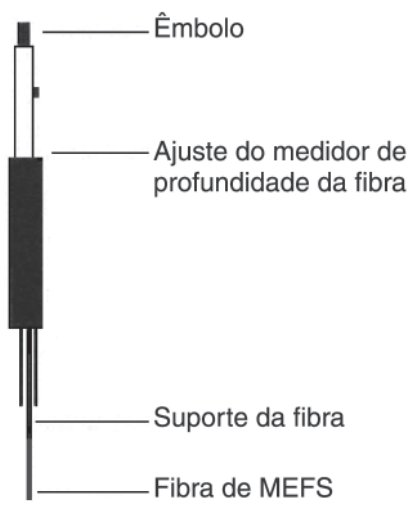

Figura 2. Dispositivos de SPME manual da SUPELCO

Os diferentes tipos de fibras utilizados com o dispositivo mostrado na Figura 2 são produzidas por dois processos, sendo um para obtenção de fibras para fases homogêneas e o outro, para fases heterogêneas. No processo para fases homogêneas, algumas fibras são obtidas por meio de cabos de fibras ópticas. Através do cabo da fibra óptica, uma haste de sílica fundida é preparada por aquecimento numa espessura de $110 \pm 5 \mu \mathrm{m}$. A fibra obtida é resfriada à temperatura ambiente e posteriormente conduzida para um aplicador contendo a solução que constituirá a fase de coleta. A ligação da fase de coleta com a fibra ocorre quando a solução da fase polimérica é depositada na fibra. O polímero presente na fibra é seco por exposição à temperatura controlada ou luz ultravioleta. O diâmetro da haste de sílica fundida e a espessura da fase de cole- ta são controlados por computador através de um comparador óptico Micro Vu Spectra 14, o que permite obter fibras reprodutíveis. As três fibras comerciais de polidimetilsiloxano (PDMS) e a fibra de poliacrilato (PA) disponíveis no mercado são fabricadas de acordo com este processo ${ }^{14}$. $\mathrm{O}$ outro processo para produção das fibras contendo fases heterogêneas, como a PDMS/divinilbenzeno (DVB), carboxen/PDMS, carbowax/DVB e carbowax/resina TPR-100 suportada (TPR) é muito complexo e ainda não foi descrito completamente ${ }^{14}$

A espessura da fase de coleta de uma fibra de SPME é determinada através da diferença entre o raio da haste de sílica fundida e o raio da fibra de coleta:

$\mu_{\mathrm{fe}}=\frac{\mathrm{d}_{\mathrm{fe}}-\mathrm{d}_{\mathrm{fsf}}}{2}$

onde: $\mathrm{m}_{\mathrm{fe}}=$ espessura da fase para coleta; $\mathrm{d}_{\mathrm{fe}}=$ diâmetro da fase de coleta e $d_{\text {fsf }}=$ diâmetro da fibra de sílica fundida

As fibras são condicionadas em temperaturas específicas em atmosfera inerte e testadas com soluções padrão para avaliar a reprodutibilidade. Elas são cortadas individualmente no comprimento de $2 \mathrm{~cm}$ e, em seguida, $1 \mathrm{~cm}$ da fase de coleta presente na fibra é retirada e então fixada na agulha da seringa através de processo químico (cola epóxi) ou físico. Desta forma, o comprimento da fibra que será exposta para amostragem é de $1 \mathrm{~cm}$ e o volume pode ser calculado pela seguinte fórmula:

$\mathrm{V}_{\mathrm{fe}}=\mathrm{V}_{\mathrm{tf}}-\mathrm{V}_{\mathrm{fsf}}$

onde: $\mathrm{V}_{\mathrm{fe}}=$ volume da fase para coleta; $\mathrm{V}_{\mathrm{tf}}=$ volume total da fibra $\mathrm{e}$ $\mathrm{V}_{\mathrm{fsf}}=$ volume da fibra de sílica fundida

O volume total da fibra ou o volume da haste da fibra de sílica fundida podem ser calculados usando a fórmula abaixo:

$\mathrm{V}=\pi \mathrm{r}^{2} \mathrm{~h}$

onde:

$\mathrm{V}=$ volume total da fibra ou da haste de sílica fundida

$\mathrm{r}=$ raio total da fibra ou da haste de sílica fundida

$\mathrm{h}=$ comprimento da fibra e da haste

Os principais tipos de fibra disponíveis no mercado e seus respectivos volumes, espessura de fase e outras informações estão descritos na Tabela 1 .

A fibra de PDMS de $7 \mu \mathrm{m}$ contém ligação entrecruzada, que é produzida através de uma reação catalítica. Estas fibras possuem alta estabilidade térmica quando comparadas com as fibras não ligadas. A estabilidade térmica da fibra é determinada pela habilidade da fase entrecruzar com ela mesmo e ligar-se com a própria sílica fundida. A introdução de grupo vinil fornece à fase maior habilidade para entrecruzar, constituindo fibras mais estáveis. A fase de poliacrilato é parcialmente entrecruzada e altamente polar.

As fibras porosas são cobertas com vários polímeros porosos e misturadas com fases poliméricas parcialmente entrecruzadas. Geralmente, estas fibras têm baixa estabilidade mecânica em relação às fases poliméricas homogêneas, porém possuem alta seletividade. Os seguintes resultados positivos são obtidos quando se aumenta a porosidade das partículas do polímero presente na fibra: aumento da capacidade total da fibra; adsorção mais forte do analito na fase e aumento da seletividade da fibra ${ }^{14}$. A fibra de PDMS/DVB foi introduzida em 1996 e refere-se a uma mistura de polímeros sólidos porosos de DVB com líquido polimérico PDMS. A área superficial desta fibra é de aproximadamente $750 \mathrm{~m}^{2} / \mathrm{g}$ constituída de uma superfície mesoporosa, com alguns macro e microporos, cuja porosidade 
Tabela 1. Algumas características das fibras de SPME disponíveis comercialmente para amostragem

\begin{tabular}{|c|c|c|c|c|c|}
\hline Fase Estacionária & Espessura $\mu \mathrm{m}$ & Ligação Química $^{1}$ & Temperatura ${ }^{2}\left({ }^{\circ} \mathrm{C}\right)$ & Volume $(\mu \mathrm{L})$ & Uso recomendado \\
\hline \multirow[t]{3}{*}{ PDMS } & 100 & Não & $200-270$ & 0,612 & $\mathrm{COV}$ \\
\hline & 30 & Não & $200-270$ & 0,132 & COSV apolares \\
\hline & 7 & Sim & $220-320$ & 0,026 & COSV semi-polares \\
\hline \multirow[t]{2}{*}{ PDMS/DVB } & 65 & Parcialmente entrecruzada & $200-270$ & 0,357 & Voláteis polares \\
\hline & 60 & Parcialmente entrecruzada & $200-270$ & 0,415 & CLAE \\
\hline CX/ PDMS & 75 & Parcialmente entrecruzada & $240-300$ & 0,436 & COV nível de traço \\
\hline CW/DVB & 65 & Parcialmente entrecruzada & $200-260$ & 0,357 & Compostos polares \\
\hline StableFlex & $50 / 30$ & Altamente entrecruzada & $230-270$ & $0,70 / 0,14$ & \\
\hline \multicolumn{6}{|l|}{ DVB/CX/PDMS } \\
\hline StableFlex & $50 / 30 *$ & Altamente entrecruzada & $230-270$ & $\sim 1,40 / 0,28$ & - \\
\hline \multicolumn{6}{|l|}{ DVB/CX/PDMS } \\
\hline $\mathrm{CW} / \mathrm{TPR}^{3}$ & 50 & Parcialmente entrecruzada & - & 0,330 & Compostos polares \\
\hline PA & 85 & Parcialmente entrecruzada & $220-310$ & 0,616 & COSV polares \\
\hline
\end{tabular}

CW: carbowax; CX: carboxen; DVB: divinilbenzeno; PA: poliacrilato; PDMS: polidimetilsiloxano. TPR: resina suportada. * Comprimento da fibra $2 \mathrm{~cm} ; 1$ - Presença de ligação química entre a fase de extração e a fibra de sílica fundida; 2 - Temperaura recomendada para operação; 3 - Não contém cola epóxi. Adaptada das ref. 14 e 15.

tem um alto grau, cerca de $1,5 \mathrm{~mL} \mathrm{~g}^{-1}$. Os microporos são regularmente maiores que os microporos das partículas de carboxen, com um diâmetro médio de $17 \AA$. A fibra contendo CW/DVB é uma mistura de partículas de polímero poroso sólido divinilbenzeno com a fase líquida carbowax. Esta fase é mais polar que a fase PDMS/DVB e é altamente sensível a oxigênio em temperaturas elevadas, devido à possibilidade de reação de oxidação. $\mathrm{O}$ resultado do ataque do oxigênio é uma fibra escura e com presença de partículas muito finas (pó). O procedimento para aumentar o tempo de vida da fibra é colocar purificadores catalíticos no gás de arraste utilizado em cromatografia. A fibra PDMS/CX, que foi introduzida no mercado em 1997, é o resultado da mistura de partículas de carboxen poroso 1006 na faixa de 1-5 $\mu \mathrm{m}$ com o polímero líquido PDMS. Esta fibra possui um volume total dos poros de $0,78 \mathrm{~mL} / \mathrm{g}$ e diâmetro dos poros na faixa de 2-20 ̊. Desta forma, a fibra de PDMS/DVB é ideal para moléculas na faixa de $\mathrm{C}_{2}-\mathrm{C}_{12}$. Moléculas maiores que $\mathrm{C}_{12}$ serão fortememte retidas na superfície da partícula e será difícil dessorvêlas posteriormente. A fibra CW/TPR é produzida pela mistura da resina porosa SUPELCOGEL TPR-100, a qual é constituída por polidivinilbenzeno/metacrilato com o polímero líquido carbowax. A resina TPR-100 é menos hidrofóbica que uma resina de divinilbenzeno, mas menos hidrofílica que uma resina metacrilato, esta propriedade oferece uma alta seletividade em relação as demais fibras. A preparação desta resina produz ligações entrecruzadas com tamanho médio das partículas em 5,5 $\mu \mathrm{m}$ e microporos menores que $20 \AA$.

A seleção com sucesso da fibra que será utilizada num trabalho de amostragem depende de vários fatores, de um modo geral a eficiência da absorção/adsorção e da dessorção depende dos seguintes fatores ${ }^{14}$ : peso molecular e tamanho do analito; ponto de ebulição e pressão de vapor do analito; polaridade do analito e da fibra; grupos funcionais do analito e da fibra e faixa de concentração e tipo de detector usado.

Em geral, fases mais espessas (maior volume) mostram maior capacidade de extrair os compostos orgânicos. A redução da espessura do filme de revestimento de 100 para $7 \mu \mathrm{m}$ produz uma fase mais estável a altas temperaturas e permite a análise de compostos com maiores pontos de ebulição. O processo de dessorção é também mais rápido em fibras menos espessas, devido à facilidade de transferência do analito da fibra para o injetor ${ }^{15}$.

As fibras de PDMS e PA, que têm uma fase não porosa e amorfa, são muito utilizadas para amostragem de compostos orgânicos voláteis $(\mathrm{COV})$ presentes em amostras ambientais, com objetivo de rea- lizar análise quantitativa ou qualitativa por cromatografia gasosa acoplada à espectrometria de massas, como mostra a revisão feita por Benfenati et al..$^{16}$. A facilidade de coleta e o fato de que o processo de absorção não é afetado pela competição entre os analitos absorvidos impulsionaram o desenvolvimentos de muitos métodos de amostragem utilizando a fibra de PDMS. Mas, por outro lado, as fibras de PDMS/DVB e Carboxen/PDMS são as mais sensíveis e seletivas comparadas às fibras poliméricas líquidas para coleta de $\mathrm{COV}^{11}$.

\section{FIBRAS DE SPME ABSORVENTES E ADSORVENTES}

Dependendo da fibra de SPME que está sendo utilizada, dois processos diferentes de coleta estão presentes. Um processo é a absorção e o outro, a adsorção. Fibras absorventes extraem os analitos pelo processo de partição dentro de uma fase líquida. $\mathrm{O}$ processo de partição é baseado na distribuição do analito entre duas fases imiscíveis, que é determinado pela fugacidade de um composto em cada fase e, em equilíbrio pode ser descrito através de uma constante dimensional (coeficiente de partição - K):

$\mathrm{K}=\frac{\mathrm{C}_{\mathrm{o}}^{\infty}}{\mathrm{C}_{\mathrm{s}}^{\infty}}$

onde: $\mathrm{C}_{\mathrm{O}}^{\infty}=$ concentração de equilíbrio de um composto da fase orgânica (ex: solvente, fibra e outros) e $\mathrm{C}_{\mathrm{S}}^{\infty}=$ concentração de equilíbrio na amostra.

A habilidade da fibra absorvente reter determinadas quantidades de analitos depende principalmente da espessura da camada líquida e do tamanho do analito. A polaridade da fibra pode aumentar a atração de determinado analito, mas é a espessura do filme líquido na fibra que retém o analito.

Fibras adsorventes extraem os analitos por interações físicas. A coleta ocorre por retenção dos analitos dentro dos poros internos. Os micro e mesoporos são ideais para analitos pequenos e intermediários e, normalmente os analitos ficarão retidos até que seja feita uma dessorção térmica ou por solventes. Macroporos podem adsorver grandes analitos através de interações de Van der Waals ou pontes de hidrogênio. Nestes tipos de fibras há um número limitado de sítios de adsorção, portanto ocorre uma competição entre os analitos, ao contrário da fibra absorvente. O modelamento da concentração de equilíbrio de um composto na fibra e sua con- 
centração na amostra são comumente descritos por isotermas de adsorção. A isoterma de adsorção que descreve adequadamente a coleta do analito em equilíbrio através das fibras PDMS/DVB e CW/DVB é a isoterma de Langmuir ${ }^{16}$. No modelo de Langmuir, a superfície tem um número limitado de sítios de adsorção que podem ser ocupados pelo analito. O processo de adsorção é tratado como uma reação entre a molécula $(\mathrm{A})$ e o sítio vazio $(\mathrm{S})$ para produzir um analito complexo $\left(\mathrm{A}_{\mathrm{ad}}\right)^{17}$ :

$\mathrm{A}+\mathrm{S} \leftrightharpoons \mathrm{A}_{\mathrm{ad}}$

As taxas de adsorção $\left(r_{a d}\right)$ e dessorção $\left(r_{d}\right)$ são as seguintes:

$\mathrm{r}_{\mathrm{ad}}=\mathrm{K}_{\mathrm{ad}}[\mathrm{A}][\mathrm{S}]$

$\mathrm{r}_{\mathrm{d}}=\mathrm{K}_{\mathrm{d}}\left[\mathrm{A}_{\mathrm{ad}}\right]$

onde: $[\mathrm{A}]$ = concentração de A na matriz; $[\mathrm{S}]=$ concentração de sítios não ocupados na superfície da fase sólida em $\mathrm{mol} \mathrm{cm} \mathrm{cm}^{-2}$; $\left[\mathrm{A}_{\mathrm{ad}}\right]$ = concentração de A na fase sólida em $\mathrm{mol} \mathrm{cm}^{-2}$ e $\mathrm{K}_{\mathrm{ad}}$ e $\mathrm{K}_{\mathrm{d}}=$ constantes.

Em condições de equilíbrio $r_{\text {ad }}$ é igual a $r_{d}$, desta forma, o processo de adsorção é baseado $\mathrm{em}^{17}$ :

$\frac{[\mathrm{Aad}]}{[\mathrm{A} \mathrm{S}]}=\frac{\mathrm{kad}}{\mathrm{kd}}=\psi$

onde: $\psi=$ constante de equilíbrio em fase adsorvente.

Um diagrama ilustrando os processos de absorção e adsorção pode ser visto na Figura 3.

Um resumo das interações que estão presentes nas fibras disponíveis comercialmente é descrito na Tabela 2. Os dados de sílica fundida foram adicionados na tabela porque a superfície de sílica fundida também interage com os analitos através do processo de adsorção.

Em seguida, serão discutidos os modelos teóricos para estimar a quantidade de analito no ar através da determinação da concentração ou massa presente nas fibras absorvente e adsorvente.

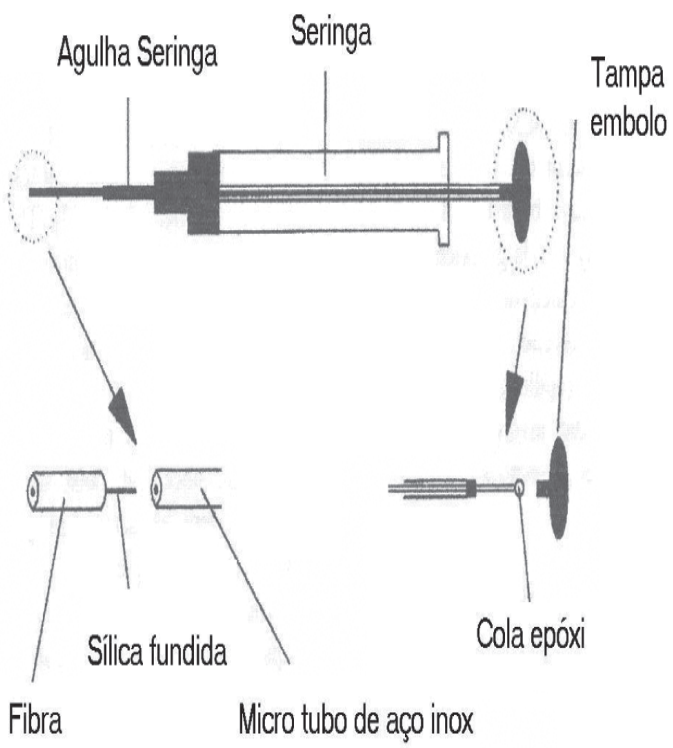

Figura 3. Comparação dos mecanismos de adsorção e absorção. Diagramas da esquerda ilustram o estágio inicial da extração e da direita, o estágio em equilíbrio. Adaptado da ref. 6
Tabela 2. Resumo das interações presentes nas fibras de SPME

\begin{tabular}{lll}
\hline Fibra de SPME & Interação & Polaridade \\
\hline Sílica fundida pura & Adsorção & Desconhecida \\
PDMS & Absorção & Apolar \\
PA & Absorção & Polar \\
PDMS/DVB & Adsorção & Mista \\
CX/ PDMS & Adsorção & Mista \\
CW/DVB & Adsorção & Polar \\
StableFlex DVB/CX/ PDMS & Adsorção & Mista \\
CW/TPR & Adsorção & Mista \\
\hline
\end{tabular}

Adaptado da ref. 39

\section{TEORIA PARA AMOSTRAGEM COM A FIBRA EXPOSTA}

Entende-se por amostragem com a fibra exposta, o processo que ocorre quando a fibra de SPME se encontra exposta na parte externa da agulha.

\section{Processo de absorção}

Em condição de equilíbrio, a quantidade de analito extraída pela fibra de SPME depende do coeficiente de partição entre a fibra e a amostra $\left(\mathrm{K}_{\mathrm{fs}}\right)$, dos volumes da fibra e da amostra de ar e, finalmente, da concentração do analito na amostra de ar como descrito por Pawliszyn ${ }^{18}$. A Equação 1 apresenta as condições de equilíbrio quando a fibra é constituída de uma fase polimérica líquida como PDMS.

$\mathrm{m}=\frac{\mathrm{K}_{\mathrm{fs}} \mathrm{V}_{\mathrm{f}} \mathrm{C}_{\mathrm{o}} \mathrm{V}_{\mathrm{s}}}{\mathrm{K}_{\mathrm{fs}} \mathrm{V}_{\mathrm{f}}+\mathrm{V}_{\mathrm{s}}}$

onde: $\mathrm{m}=$ massa do analito absorvida pela cobertura; $\mathrm{V}_{\mathrm{f}}$ e $\mathrm{V}_{\mathrm{s}}=$ volumes da fase líquida e da amostra de ar, respectivamente; $\mathrm{K}_{\mathrm{fs}}=$ coeficiente de partição do analito entre a fase e a amostra e $\mathrm{C}_{\mathrm{o}}=$ concentração inicial do analito na amostra.

Esta equação indica uma relação linear entre a quantidade de analito absorvida pelo revestimento da fibra e a concentração inicial do analito na amostra. Esta relação é fundamental para a quantificação de analitos presentes na fibra .

Segundo Pawliszyn ${ }^{18}$ quando o volume de amostra é muito grande $\left(\mathrm{Vs}>>\mathrm{K}_{\mathrm{fs}} \mathrm{V}_{\mathrm{f}}\right)$, o sistema pode ser descrito pela Equação 2, onde a quantidade de analito $(\mathrm{m})$ absorvido pela fibra é proporcional ao coeficiente de partição fibra/amostra $\left(\mathrm{K}_{\mathrm{fs}}\right)$, volume da fibra $\left(\mathrm{V}_{\mathrm{f}}\right)$ e concentração inicial do analito na amostra $\left(\mathrm{C}_{\mathrm{o}}\right)$.

$\mathrm{m}=\mathrm{K}_{\mathrm{fs}} \mathrm{V}_{\mathrm{f}} \mathrm{C}_{\mathrm{o}}$

Para obter a concentração do analito no ar em determinada temperatura $^{19}$, a Equação 2 pode ser rearranjada na Equação 3.

$\mathrm{C}_{\mathrm{ar}}=\frac{\mathrm{C}_{\mathrm{f}}}{\mathrm{K}}=\frac{\mathrm{m}_{\mathrm{f}}}{\mathrm{V}_{\mathrm{f}}} \frac{1}{\mathrm{~K}}$ (em determinada temperatura $)$

onde: $\mathrm{C}_{\mathrm{ar}}$ e $\mathrm{C}_{\mathrm{f}}=$ concentrações dos analitos no ar e na fibra, respectivamente, na unidade de $\mathrm{mg} / \mathrm{m}^{3} ; \mathrm{K}=$ coeficiente de partição do analito entre a fibra e o ar; $\mathrm{m}_{\mathrm{f}}=$ massa do analito na fibra $(\mathrm{mg}) \mathrm{e}$ $\mathrm{V}_{\mathrm{f}}=$ volume da fibra. $\left(\mathrm{m}^{3}\right)$.

$\mathrm{O}$ conhecimento do valor de $\mathrm{K}$ na Equação 3 é fundamental para amostragem do ar com a fibra PDMS. Quando uma amostragem do ar é realizada, o valor $\mathrm{m}_{\mathrm{f}}$ pode ser determinado experimental- 
mente, se $V_{f}$ e $K$ são conhecidos. Quando a temperatura de amostragem é diferente da temperatura na qual $\mathrm{K}$ foi calculado, uma relação linear entre $\mathrm{K}$ e a temperatura pode ser usada para corrigir o valor de K, como mostra a Equação $4^{20}$.

$\log \mathrm{K}=\frac{\Delta \mathrm{H}^{\mathrm{v}}}{2,303 \mathrm{RT}}+\left[\log \left(\frac{\mathrm{RT}}{\gamma_{\mathrm{i}} \rho^{*}}\right)\right]-\frac{\Delta \mathrm{H}^{\mathrm{v}}}{2,303 \mathrm{RT}^{*}}$

onde: $\Delta \mathrm{H}^{\mathrm{v}}=$ calor de vaporização do analito $(\mathrm{kJ} / \mathrm{mol}) ; \mathrm{R}=$ constante dos gases $\left(\mathrm{J} / \mathrm{K}^{*} \mathrm{~mol}\right) ; \mathrm{T}=$ temperatura de amostragem $(\mathrm{K}) ; \rho^{*}=$ pressão de vapor do soluto em uma temperatura conhecida (atm); $\mathrm{T}^{*}=$ temperatura do soluto em uma temperatura conhecida $(\mathrm{K})$ e $\gamma_{\mathrm{i}}$ $=$ coeficiente de atividade do soluto.

A Equação 4 pode ser simplificada da seguinte forma:

$\log K=\frac{a}{T}+b$

onde: $\mathrm{a}=\frac{\Delta \mathrm{H}^{\mathrm{v}}}{2,303 \mathrm{R}}$ e $\mathrm{b}=\left[\log \left(\frac{\mathrm{RT}}{\gamma_{\mathrm{i}} \mathrm{p}^{*}}\right)\right]-\frac{\Delta \mathrm{H}^{\mathrm{v}}}{2,303 \mathrm{R} \mathrm{T}^{*}}$

Substituindo a Equação 5 em 3 obtém-se a Equação $6^{20}$ :

$\mathrm{C}_{\mathrm{ar}}=\frac{\mathrm{m}_{\mathrm{f}}}{\mathrm{V}_{\mathrm{f}} \times 10^{-\left(\frac{\mathrm{a}}{\mathrm{T}}+\mathrm{b}\right)}}$

Utilizando a Equação 6, a concentração de compostos no ar na unidade de $\mathrm{mg} / \mathrm{m}^{3}$ pode ser obtida quando não se conhece o valor de $\mathrm{K}$ para a temperatura na qual foi realizada a amostragem. As Equações 3 e 6 não dependem do volume da amostra, sendo assim a amostragem em campo por SPME não exige coleta do ar em um volume conhecido de amostra. A fibra pode ser exposta diretamente ao ar de uma sala ou ambiente externo.

A Equação 6 pode ser simplificada para fornecer a concentração atmosférica de analitos na unidade de partes por milhão volume por volume $\left(\mathrm{ppm}_{\mathrm{v}}\right)$. A Equação 7 foi deduzida por Martos e Pawliszyn ${ }^{20}$ e sua aplicação exige apenas que se faça a determinação da massa de analito presente na fibra de PDMS e que se determine os valores apropriados de $\alpha$ e $\beta$ para uma determinada fibra, em uma temperatura específica.

$\mathrm{C}=(\mathrm{mg}$ na fibra $) \alpha \beta$

onde: $\mathrm{C}=$ concentração do analito no ar em $\mathrm{ppm}_{\mathrm{v}} ; \alpha=\frac{R}{M M P V_{f}}$ onde, $\mathrm{R}=$ constante dos gases, $\mathrm{MM}=$ massa molecular, $\mathrm{P}=$ pressão atmosférica, $\mathrm{Vf}=$ volume da fibra; $\beta=\frac{\mathrm{T}}{10^{\left(\left(\frac{\mathrm{a}}{\mathrm{T}}\right)+\mathrm{b}\right)}}$ onde, $\mathrm{T}=$ temperatura.

É possível substituir $\beta$ por [T/K] na Equação 7 quando o valor de $\mathrm{K}$ do analito foi determinado na mesma temperatura daquela em que ocorreu a amostragem. Uma margem de erro tolerável é introduzida quando se ignora a dependência da temperatura de amostragem para os valores de K numa faixa estreita de 296-300 K. O uso de valores de $\mathrm{K}$ determinados em $298 \mathrm{~K}$ para análise quantitativa na faixa de 296-300 K não é recomendado, mas pode ser usado para estimar a concentração ${ }^{20}$.

Martos et al. ${ }^{19}$ propuseram um modelo para estimar o coeficiente de distribuição (K) entre a fibra de PDMS e o ar, utilizando o sistema de índice de tempo de retenção programado linear (LTPRI), o qual é usado em cromatografia para identificar compostos em cromatogramas usando apenas informações do seu tempo de re- tenção. Esta alternativa aplicada à técnica de SPME simplifica a amostragem de compostos, visto que não há necessidade de calibração da fibra. A Equação 8 é utilizada para obter os valores de K para uma série homóloga de átomos de carbono.

$\log \mathrm{K}=\mathrm{a}+\mathrm{b}($ LTPRI)

Este modelo foi aplicado para determinar o valor de $\mathrm{K}$ teórico para 29 compostos isoparafínicos e 33 compostos aromáticos. Para realizar esta determinação, foram construídas curvas analíticas Log x LTPRI para pentano, hexano, heptano, octano, nonano, decano, undecano, dodecano, tridecano e tetradecano. Os valores de K para os referidos compostos foram obtidos experimentalmente pela equação 3 e os valores para LTPRI obtidos através da equação 9 ou através de base de dados como Sadtler ${ }^{21}$. Os valores de K teórico calculados pelo método LTPRI foram comparados com os valores de $\mathrm{K}$ obtidos experimentalmente. $\mathrm{O}$ resultado mostrou excelente correlação entre os métodos.

LTPRI $=100 \times\left(\frac{t_{r(A)}-t_{r(n)}}{t_{r(n+1)}-t_{r(n)}}\right)+100 n$

onde: $\mathrm{t}_{\mathrm{r}(\mathrm{A})}=$ tempo de retenção do analito; $\mathrm{t}_{\mathrm{r}(\mathrm{n})}=$ tempo de retenção do $n$-alcano que elui antes do $\operatorname{tr}(\mathrm{A}) ; \mathrm{t}_{\mathrm{r}(\mathrm{n}+1)}=$ tempo de retenção do $\mathrm{n}$ alcano que elui depois do $\operatorname{tr}(\mathrm{A})$ e $\mathrm{n}=$ número de átomos de carbono para $\operatorname{tr}(\mathrm{n})$.

No trabalho apresentado por Martos e Pawliszyn ${ }^{20}$, os autores propuseram um modelo onde o sistema está num estado de equilíbrio, ou seja, a $\mathrm{C}_{\text {fibra }}$ não muda com o tempo, entretanto, a massa depositada na fibra pode variar em diferentes valores de fluxo e umidade e, portanto, uma situação de não equilíbrio pode estar presente. Esta situação foi investigada apenas experimentalmente por Martos e Pawliszyn, porém não foi considerada nos modelos propostos pelos autores.

Um modelo para calcular a concentração de compostos no ar com a fibra de SPME/PDMS em condições de não equilíbrio, considerando a presença de fluxo, foi proposto por Barlelt e Zilkowski ${ }^{10}$. A Equação 10 permite obter o valor de concentração absoluto do analito no ar a partir da determinação da massa presente na fibra, independente se o equilíbrio foi atingido ou não.

$\frac{\mathrm{M}_{\text {fibra }}}{\mathrm{C}_{\mathrm{ar}}}=\mathrm{K}^{*}\left(1-\exp \left(\frac{-\mathrm{AF} \mathrm{t}}{\mathrm{K}^{*}}\right)\right)$

onde: $\mathrm{M}_{\text {fibra }}=$ massa do analito na fibra (ng); $\mathrm{C}_{\mathrm{ar}}=$ concentração no $\operatorname{ar}(\mathrm{ng} / \mathrm{mL}) ; \mathrm{F}=$ fluxo de $\operatorname{ar}(\mathrm{mL} / \mathrm{min}) ; \mathrm{A}=$ fração de analitos no fluxo de ar que interage com a fibra num determinado período; $\mathrm{t}=$ tempo de amostragem (min) e $\mathrm{K}^{*}=$ coeficiente de partição calculado conforme Martos ${ }^{19}$.

Os mesmos autores que propuseram o modelo da Equação 10 apresentaram, posteriormente, modelos para cálculo de $\mathrm{K}^{*}$ e A para serem utilizados em situações com fluxo de ar na faixa de 2-220 $\mathrm{mL} / \mathrm{min}$. Os valores de $\mathrm{K}^{*}$ e A, segundo o modelo de Bartelt e Zilkowski ${ }^{22}$, podem ser obtidos pelas Equações 11, 12 e 13:

$\log K^{*}=-3,5340+G+(0,005062-0,00002853 \times$ TEMP $) \times$ LTPRI

onde, TEMP $=$ temperatura de amostragem em ${ }^{\circ} \mathrm{C}$; LTPRI $=$ índice de retenção programado em temperatura linear numa coluna apolar; $\mathrm{G}=$ constante que depende somente do grupo funcional $(\mathrm{G}=0,000$ para hidrocarbonetos, $\mathrm{G}=0,100$ para éster metílico e $\mathrm{G}=0,360$ para álcoois). 
$\mathrm{A}=1-0,00199 \times \mathrm{F}^{3,08}$

$\mathrm{A}=2,34 \times \mathrm{F}^{-1,715}$

se $\mathrm{F} \leq 4,4 \mathrm{~mL} / \mathrm{min}$

se $\mathrm{F}>4,4 \mathrm{~mL} / \mathrm{min}$

onde, $\mathrm{F}$ = fluxo do ar.

Na revisão bibliográfica realizada não se encontrou um modelo teórico que considerasse o valor de umidade do local de amostragem diretamente no cálculo da concentração de analitos no ar. Então, a alternativa encontrada por Gorlo et al. ${ }^{23}$ e Namiesnik et al..$^{24}$ foi realizar a quantificação de analitos no ar utilizando um sistema de calibração onde a temperatura, velocidade e umidade do ar durante a calibração eram próximas ao local de amostragem. A utilização da mesma fibra para coleta dos analitos em amostras e em câmaras para calibração diminuem os erros relacionados à fibra, além de poder gerar condições atmosféricas o mais próximo possível da amostra ${ }^{25}$. O procedimento de quantificação experimental tem sido utilizado por muitos autores, como Chai et al. ${ }^{12,26}$, Gorlo et al. ${ }^{23,29}$, Grote e Pawliszyn ${ }^{27}$, Bolta et al. ${ }^{28}$, Namiesnik ${ }^{24}$ e Martos $^{30}$.

A teoria acima não se aplica quando a amostragem por SPME é realizada com a seringa recolhida dentro da agulha, pois neste caso o processo predominante de transporte de analitos da fase gasosa para a fibra ocorre por difusão ${ }^{18}$.

\section{Processo de adsorção}

Fibras poliméricas, tais como PDMS/DVB, CW/DVB e CX/ PDMS possuem número limitado de sítios ativos na superfície, portanto, a quantidade de analitos extraídos será proporcional ao número de sítios. Por esta razão, a relação entre a quantidade de analito extraído e sua concentração na amostra não é linear em altas concentrações. Adsorção é um processo competitivo no qual moléculas com baixa afinidade pela fibra podem ser substituídas por aquelas de alta afinidade. Desta forma, a descrição teórica da coleta baseada em adsorção difere daquela que governa a absorção ${ }^{18}$.

Quando a fibra é exposta ao ar em movimento, uma interface de espessura $\delta$ desenvolve-se entre o ar e a superfície da fibra. Os analitos são transferidos do ar para a superfície da fibra via difusão molecular, como mostra a Figura 4. Em muitos casos, a difusão molecular de analitos pela interface é a etapa determinante do processo de adsorção.

A concentração dos analitos no ar $\left(\mathrm{C}_{\mathrm{g}}\right)$ pode ser considerada constante quando há um curto período de amostragem e um fornecimento constante de analito por convecção. Estas suposições são verdadeiras para muitos casos de amostragem de ar por SPME,

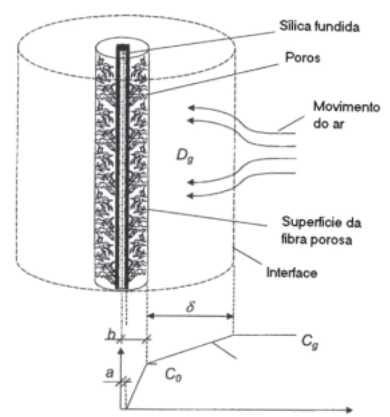

Figura 4. Processo de extração com SPME utilizando fibras porosas. $D_{g}=$ coeficiente de difusão molecular na fase gasosa $\left(\mathrm{cm}^{2} / \mathrm{s}\right) ; b=$ raio externo da fibra (cm); a=diâmetro interno da fibra $(\mathrm{cm}) ; C_{g}=$ concentração do analito no $\operatorname{ar}(\mathrm{ng} / \mathrm{mL}) ; C_{o}=$ concentração na superfície da fibra $(\mathrm{ng} / \mathrm{mL}) ; \delta=$ espessura da interface (cm). Adaptado da ref. 11 onde o volume de ar é muito maior que o volume da interface e o processo de coleta não afeta a concentração do analito no ar. O comportamento da concentração do analito na fase gasosa até a superfície da fibra pode ser linear, como mostrado no pequeno gráfico presente na Figura 4. Além disto, a concentração na superfície da fibra pode ser considerada igual a zero quando o processo de coleta é iniciado. O processo de difusão dentro dos poros de um sólido é o princípio que controla a transferência de massa de analitos de $b$ (diâmetro externo da fibra) até $a$ (diâmetro interno da fibra). Com base no princípio de transferência de calor dentro de um cilindro de diâmetros interno e externo b e d e de outras informações, Koziel et al. ${ }^{11}$ propuseram a Equação 14 para calcular a massa de analito extraída pela fibra em relação ao tempo de amostragem

$\mathrm{m}_{(\mathrm{t})}=\frac{2 \pi \mathrm{D}_{\mathrm{g}} \mathrm{L}}{\left(\frac{\ln (\mathrm{b}+\delta)}{\mathrm{b}}\right)} \int_{0}^{\mathrm{t}} \mathrm{C}_{\mathrm{g}}(\mathrm{t}) \mathrm{dt}$

onde: $\mathrm{m}=$ massa de analito extraído sobre o tempo de amostragem (ng); $\mathrm{t}=$ tempo de amostragem (s); $\mathrm{D}_{\mathrm{g}}=$ coeficiente de difusão molecular na fase gasosa $\left(\mathrm{cm}^{2} / \mathrm{s}\right) ; \mathrm{b}=$ raio externo da fibra $(\mathrm{cm}) ; \mathrm{L}$ = comprimento da fibra $(\mathrm{cm}) ; \mathrm{C}_{\mathrm{g}}=$ concentração do analito no ar $(\mathrm{ng} / \mathrm{mL})$ e $\delta=$ espessura da interface $(\mathrm{cm})$.

Considerando que a concentração do analito é constante por um tempo de amostragem muito pequeno, então a Equação 14 pode ser reduzida à Equação $15^{11}$ :

$\mathrm{m}_{(\mathrm{t})}=\frac{2 \pi \mathrm{D}_{\mathrm{g}} \mathrm{L}}{\left(\frac{\ln (\mathrm{b}+\delta)}{\mathrm{b}}\right)} \mathrm{C}_{\mathrm{g}}(\mathrm{t})$

O comprimento e o diâmetro externo da fibra são constantes para cada tipo comercializado. O comprimento das fibras PDMS/ DVB $65 \mu \mathrm{m}$ e PDMS/CX $75 \mu \mathrm{m}$ é $1,0 \mathrm{~cm}$ e o diâmetro externo (2b) é $0,0240 \mathrm{~cm}( \pm 10 \%)$ e $0,0260 \mathrm{~cm}( \pm 10 \%)$, respectivamente ${ }^{11}$. Para se obter o valor da concentração do analito no ar em situações de amostragem rápida com SPME, a Equação 15 transforma-se em:

$\mathrm{C}_{\mathrm{g}}=\frac{\mathrm{m}(\mathrm{t}) \ln \left(\frac{\mathrm{b}+\delta}{\mathrm{b}}\right)}{2 \pi \mathrm{D}_{\mathrm{g}} \mathrm{Lt}}$

Quando a espessura da interface é muito menor que o raio externo da fibra $\delta<$ b, a Equação 15 transforma-se em Equação $17^{11}$, que é semelhante à equação para cálculo da massa de analito na fibra no processo de amostragem com a fibra recolhida, sendo que $\mathrm{Z}$ é substituído por $\delta^{15}$.

$\mathrm{m}(\mathrm{t})=\left(\frac{\mathrm{D}_{\mathrm{g}} \mathrm{A}}{\delta}\right) \mathrm{C}_{\mathrm{g}} \mathrm{t}$

Os valores de $\mathrm{D}_{\mathrm{g}}$ para cada analito podem ser encontrados na literatura ou calculados. A espessura da camada da interface $(\delta)$ pode ser estimada usando a Equação 18, que foi adaptada da teoria de transferência de calor para a fibra de SPME na presença de um fluxo cruzado ${ }^{11}$ :

$\delta=9,52\left(\frac{\mathrm{b}}{\operatorname{Re}^{0,62} \mathrm{Sc}^{0,38}}\right)$

onde: $\mathrm{Re}=$ número de Reynolds, que é igual a $2 \mathrm{ub} / \mathrm{v}$, onde u é a velocidade linear do ar $(\mathrm{cm} / \mathrm{s})$ e v é a viscosidade cinemática do ar $\left(\mathrm{cm}^{2} / \mathrm{s}\right)$ e $\mathrm{Sc}=$ número de $\mathrm{Schmidt}$, que é igual a $\mathrm{v} / \mathrm{D}_{\mathrm{g}}$. 
A outra possibilidade para quantificação de compostos amostrados por SPME utilizando fibras adsorventes é através da calibração laboratorial, ou seja através de padronização externa ${ }^{27,31-35}$.

Na revisão bibliográfica sobre fibras adsorventes não se encontrou uma descrição de modelo teórico que considerasse o valor de umidade do local de amostragem diretamente no cálculo da concentração de analitos no ar.

\section{TEORIA PARA AMOSTRAGEM COM A FIBRA PROTEGIDA}

Entende-se por amostragem com a fibra protegida, o processo que ocorre quando a fibra de SPME se encontra na parte interna da agulha.

\section{Modo ativo}

No modo ativo assume-se que o sistema de SPME consiste de dois dispositivos. O primeiro consiste de um pedaço de sílica fundida internamente coberta com uma camada de filme, tal como um pedaço de coluna capilar tubular aberta utilizada em cromatografia gasosa. No outro dispositivo de amostragem o capilar está recheado com uma fase de coleta dispersa no material de suporte, tal como um pedaço de coluna capilar utilizada em micro cromatografia líquida. Nestes arranjos geométricos, o perfil da concentração ao longo do eixo $\mathrm{x}$ do tubo em função do tempo (t) pode ser descrito pela Eequação de dispersão frontal da concentração ${ }^{15}$ :

$C(x, t)=\frac{1}{2} C_{0}\left[1-e r f \frac{\left(\frac{x-\mu t}{1+K_{p}}\right)}{\sigma \sqrt{2}}\right]$

onde: $\mathrm{t}=$ tempo $(\mathrm{s}) ; \mathrm{C}_{\mathrm{o}}=$ Concentração na fibra; $\mathrm{x}=$ comprimento do tubo $(\mathrm{cm}) ; \mu=$ velocidade linear do fluído através do tubo $(\mathrm{cm} / \mathrm{s}) ;$ erf= error function (função de erro). $\operatorname{erf}(\mathrm{x})=\frac{2}{\sqrt{\pi}} \int_{0}^{\mathrm{x}} \exp \left(-\mathrm{t}^{2}\right)$ e $K_{p}$ é a relação de partição definidas como:

$\mathrm{K}_{\mathrm{p}}=\mathrm{K}_{\mathrm{fs}} \frac{\mathrm{V}_{\mathrm{f}}}{\mathrm{V}_{\mathrm{v}}}=4 \mathrm{~K}_{\mathrm{fs}} \frac{\mathrm{d}_{\mathrm{s}}}{\mathrm{d}_{\mathrm{c}}}$, onde $\mathrm{K}_{\mathrm{fs}}$ é uma constante de distribuição amostra/fase, $V_{f}$ é o volume da fase extratora, $V_{v}$ é o volume morto do tubo contendo a fase extratora, $\mathrm{d}_{\mathrm{s}}$ é a espessura da fase estacionária e $\mathrm{d}_{\mathrm{c}}$ é o diâmetro dos poros.

$\sigma=$ raiz quadrada da dispersão frontal, calculada pela seguinte relação: $\sigma=\sqrt{\mathrm{H}_{\mathrm{t}} \frac{\mu}{1+\mathrm{K}_{\mathrm{p}}}}$, onde $\mathrm{H}$ é equivalente a altura de um prato teórico em sistemas cromatográficos.

A Equação (19) indica que os analitos migram através do capilar com uma velocidade proporcional à velocidade linear da amostra, e inversamente proporcional a partição. Para pequenos capilares com uma pequena dispersão, o tempo de coleta pode ser considerado similar ao tempo necessário para o centro da banda atingir o fim do capilar ${ }^{15}$ :

$\mathrm{t}_{\mathrm{e}}=\frac{\mathrm{L}\left(1+\mathrm{k}_{\mathrm{fs}} \frac{\mathrm{V}_{\mathrm{f}}}{\mathrm{V}_{\mathrm{v}}}\right)}{\mu}$

onde: $\mathrm{t}_{\mathrm{e}}=$ tempo de coleta (s); $\mathrm{L}=$ comprimento do capilar da fase de coleta $(\mathrm{cm}) ; \mathrm{V}_{\mathrm{f}}=$ volume da fibra $(\mu \mathrm{L}) ; \mathrm{V}_{\mathrm{v}}=$ volume morto $(\mu \mathrm{L})$ e $\mu=$ velocidade linear do fluído através do tubo $(\mathrm{cm} / \mathrm{s})$;

Como esperado, o tempo de coleta é diretamente proporcional ao comprimento do capilar e inversamente proporcional ao fluxo linear de gases. Para pequenos capilares com uma pequena dispersão, o tempo de coleta também aumenta com um aumento na constante de distribuição fase/amostra e com o volume da fase de coleta, mas diminui com um aumento no volume morto do capilar. Deve ser enfatizado que a Equação 20 pode ser usada somente para coleta direta quando a matriz passa pelo capilar. Esta técnica é limitada a amostras de gases livres de partículas. A dessorção em geral ocorre quando a fase é colocada no injetor numa temperatura alta. O tempo de dessorção corresponde à eluição de 2 volumes mortos do capilar ${ }^{15}$.

\section{Modo passivo}

Uma amostragem integrada é possível com um sistema de SPME, no modo passivo. Este modo de amostragem é particularmente importante em medidas de campo, quando ocorrem mudanças de concentração do analito ao longo do tempo.

Quando a fase de coleta não está exposta diretamente ao ar, mas está contida dentro da agulha sem nenhum fluxo de amostra presente, a coleta ocorre através da fase gasosa estática presente na agulha. Este arranjo geométrico representa um poderoso método para gerar resposta proporcional à concentração do analito em relação ao tempo e espaço. Neste caso, o único mecanismo de transporte de analito para a fase de coleta é a difusão através da fase gasosa presente no tubo entre a pequena abertura da agulha, caracterizado pela área (A) e a posição da fase sólida, localizada a uma distância $\mathrm{Z}$ da abertura da agulha. $\mathrm{O}$ esquema deste processo de difusão pode ser visto na Figura 5.

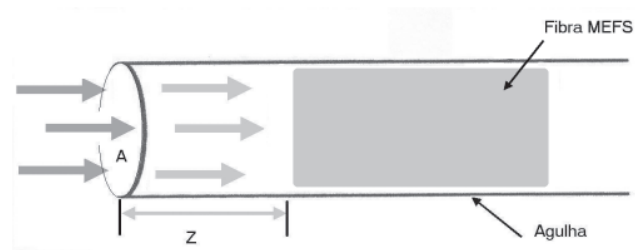

Figura 5. Esquema do processo de difusão com seringa recolhida. $A=$ área da seção transversal do percurso de difusão $\left(\mathrm{cm}^{2}\right) ; Z=$ comprimento do percurso da difusão (cm). Adaptado da ref. 37

A quantidade de analito extraído (dm), durante um intervalo de tempo (dt) pode ser calculada considerando a 1ํㅗ lei de Difusão de Fick $^{18}$.

$\mathrm{dm}=-\mathrm{A} \mathrm{D}_{\mathrm{g}} \frac{\mathrm{dc}}{\mathrm{dx}} \mathrm{dt}=-\mathrm{A} \mathrm{D}_{\mathrm{g}} \frac{\Delta \mathrm{C}(\mathrm{t})}{\mathrm{Z}} \mathrm{dt}$

onde: $\mathrm{m}=$ massa do analito $(\mathrm{g}) ; \mathrm{A}$ = área da seção transversal do percurso de difusão $\left(\mathrm{cm}^{2}\right) ; \mathrm{D}=$ coeficiente de difusão do analito no ar $\left(\mathrm{cm}^{2} / \mathrm{min}\right) ; \mathrm{Z}=$ comprimento do percurso da difusão $(\mathrm{cm})$ e $\Delta \mathrm{C}(\mathrm{t})$ é igual a $\mathrm{C}(\mathrm{t})=\mathrm{C}_{\mathrm{z}}-\mathrm{C}_{(\mathrm{t})}$, onde $\mathrm{C}_{\mathrm{z}}$ é a concentração do analito na fase gasosa nas proximidades da fibra e $\mathrm{C}(\mathrm{t})$ é a concentração do analito na fase gasosa nas proximidades da abertura da agulha.

$\mathrm{C}_{\mathrm{z}}$ é próximo de zero para uma alta constante de distribuição gás/fibra, o que leva a $\Delta \mathrm{C}(\mathrm{t})=-\mathrm{C}(\mathrm{t})$. Desta forma, a integração da Equação 21 reduz-se à Equação 22, que permite obter a massa acumulada na fibra de SPME sobre o tempo de amostragem. A Equação 22 é válida somente quando a quantidade de analito presente na fibra é uma pequena fração da quantidade em equilíbrio no ar, tipicamente menor que $5 \%$ : 
Tabela 3. Aplicações de SPME em amostragens de matrizes gasosas

\begin{tabular}{|c|c|c|c|c|c|}
\hline Matriz & Fibra & Analito & Tipo de amostragem & Análise & Ref. \\
\hline Ar ambiente & PDMS, $100 \mu \mathrm{m}$ & $\begin{array}{l}\text { Hidrocarbonetos aromáticos } \\
\text { e halogenados }\end{array}$ & $\begin{array}{l}\text { Passiva com fibra exposta } \\
\text { e recolhida }\end{array}$ & $\begin{array}{l}\text { CG-EM; CG-FID; } \\
\text { CG-DCE }\end{array}$ & 12 \\
\hline Ar ambiente & PDMS, $100 \mu \mathrm{m}$ & $\begin{array}{l}\text { Hidrocarbonetos aromáticos } \\
\text { mono e policíclicos }\end{array}$ & Passiva com fibra exposta & CG-EM & 40 \\
\hline Ar interior de apartamento & PDMS, $100 \mu \mathrm{m}$ & $\begin{array}{l}\text { Benzeno, tolueno, clorobenzeno, } \\
\text { tetracloreto de carbono, p-xileno } \\
\text { e n-decano }\end{array}$ & Passiva com fibra exposta & CG-EM & 30 \\
\hline Ar interior de laboratório químico & PDMS, $100 \mu \mathrm{m}$ & Análise qualitativa & Passiva com fibra exposta & CG-EM & 24 \\
\hline $\begin{array}{l}\text { Gás do escapamento de automóveis } \\
\text { e ar interior de residências, laboratório } \\
\text { químico e de computação e sala } \\
\text { de estudantes }\end{array}$ & $\begin{array}{l}\text { PDMS } 65 \mu \mathrm{m} \\
\text { e } 100 \mu \mathrm{m}\end{array}$ & $\begin{array}{l}\text { Formaldeído, tolueno e } \\
\text { hidrocarbonetos aromáticos } \\
\text { policíclicos }\end{array}$ & $\begin{array}{l}\text { Amostragem passiva com } \\
\text { fibra exposta }\end{array}$ & CG-EM e CG-FID & 3 \\
\hline $\begin{array}{l}\text { Ar interior de banheiro, cozinha, } \\
\text { garagem, lojas e escolas }\end{array}$ & PDMS/DVB & $\begin{array}{l}\text { Heptano, octano, nonano, } \\
\text { decano, undecano, dodecano }\end{array}$ & $\begin{array}{l}\text { Amostragem passiva com } \\
\text { fibra recolhida }\end{array}$ & CG-FID & 38 \\
\hline $\begin{array}{l}\text { Ar interior de laboratório químico, } \\
\text { sala de fumantes, trem e carro }\end{array}$ & CW/PDMS $75 \mu \mathrm{m}$ & $\begin{array}{l}\text { Benzeno, tolueno, etilbenzeno, } \\
\text { estireno e xileno }\end{array}$ & Headspace com SPME & CG-DIC & 41 \\
\hline Ar ambiente de indústria & PDMS $100 \mu \mathrm{m}$ & Análise qualitativa & Headspace com SPME & CG-EM & 42 \\
\hline Ar ambiente de indústria & PDMS $100 \mu \mathrm{m}$ & Análise qualitativa & $\begin{array}{l}\text { Amostragem passiva com } \\
\text { fibra exposta }\end{array}$ & CG-EM & 43 \\
\hline $\begin{array}{l}\text { Ar interior de lojas, casas, } \\
\text { apartamentos, escritórios e escolas }\end{array}$ & PDMS/DVB $65 \mu \mathrm{m}$ & Formaldeído & $\begin{array}{l}\text { Amostragem passiva, fibra } \\
\text { exposta e recolhida }\end{array}$ & CG-FID & 35 \\
\hline Ar ambiente de indústria & PDMS/DVB $65 \mu \mathrm{m}$ & Formaldeído & $\begin{array}{l}\text { Amostragem passiva, fibra } \\
\text { exposta e recolhida }\end{array}$ & CG-FID & 9 \\
\hline Ar expirado & $\begin{array}{l}\text { CW/DVB } 65 \mu \mathrm{m} ; \\
\text { PDMS } 100 \mu \mathrm{m} ; \\
\text { PA } 85 \mu \mathrm{m} ; \\
\text { PDMS/DVB } 65 \mu \mathrm{m}\end{array}$ & Etanol, isopreno, acetona & $\begin{array}{l}\text { Amostragem passiva com } \\
\text { fibra exposta }\end{array}$ & CG-EM & 28 \\
\hline Biogás & CX-PDMS & Compostos orgânicos de enxofre & $\begin{array}{l}\text { Amostragem ativa com } \\
\text { fibra exposta }\end{array}$ & CG-EM & 45 \\
\hline Ar interior de laboratório químico & $\begin{array}{l}\text { CX-PDMS } 75 \mu \mathrm{m} \\
\text { e PDMS } 100 \mu \mathrm{m}\end{array}$ & Diclorometano & $\begin{array}{l}\text { Amostragem passiva com } \\
\text { fibra exposta }\end{array}$ & CG-EM & 44 \\
\hline Exposição ocupacional & PDMS $100 \mu \mathrm{m}$ & Trimetilamina & $\begin{array}{l}\text { Amostragem ativa com } \\
\text { fibra exposta }\end{array}$ & CG-FID & 46 \\
\hline
\end{tabular}

$\mathrm{m}=\frac{\mathrm{D}_{\mathrm{g}} \mathrm{A}}{\mathrm{Z}} \int_{0}^{\mathrm{x}} \mathrm{C}_{(\mathrm{t})} \mathrm{dt}$

A quantidade de analito é proporcional à integral da concentração na amostra sobre o tempo, ao coeficiente de difusão do analito na fase gasosa $\left(\mathrm{D}_{\mathrm{g}}\right)$, à área de abertura da seringa $(\mathrm{A})$ e inversamente proporcional à distância da posição da fibra em relação à abertura da agulha (Z).

A taxa de amostragem (TA) de analitos pela fibra de SPME pode ser obtida teoricamente através da Equação $23^{36}$.

$\mathrm{TA}=\mathrm{D}_{\mathrm{g}} \frac{\mathrm{A}}{\mathrm{Z}}$

onde: $\mathrm{TA}=$ taxa de amostragem $\left(\mathrm{cm}^{3} / \mathrm{s}\right)$

A taxa de amostragem também pode ser obtida experimentalmente através da determinação da massa de analito presente na fibra em equilíbrio com uma concentração constante no ar utilizando a Equação 243, 9, 34, 36, 37 .
$\mathrm{TA}=\frac{\mathrm{m}}{\mathrm{C} \times \mathrm{t}}$

Os valores de TA obtidos teorica e experimentalmente utilizando fibras de PDMS e PDMS/DVB foram comparados e os valores teóricos foram mais próximos dos valores experimentais quando se utilizou a fibra PDMS/DVB. Ao comparar os dados utilizando a fibra de PDMS notou-se uma aproximação entre valores teóricos e prático somente para alguns compostos com alto peso molecular ${ }^{9,36,37}$.

Uma vez que TA tenha sido determinada experimental ou teoricamente, a concentração de analitos em amostras desconhecidas pode ser obtida simplesmente determinando-se a massa presente na fibra durante um período $t$ de amostragem (Equação 25):

$\mathrm{C}=\frac{\mathrm{m}}{\mathrm{TA} \times \mathrm{t}}$

Para tempos de amostragem maiores, as seguintes alterações 
geométricas no dispositivo de SPME podem ser realizadas: a fibra pode ser colocada mais longe da abertura da agulha (maior Z), a abertura da agulha pode ser reduzida colocando-se um orifício adicional ou uma fibra de alta capacidade pode ser utilizada. As duas primeiras alterações resultarão em baixa sensibilidade, enquanto que a última propõe melhor seletividade e sensibilidade. Uma fibra de volume maior e alta constante de distribuição fibra/analito oferece maior sensibilidade ${ }^{18}$. Para utilizar uma destas possibilidades é necessário fazer uma correção do valor da TA, caso a amostra tenha sido obtida num valor de $\mathrm{Z}$ diferente daquele obtido em laboratório. Para efetuar estas correções, utiliza-se a Equação $26^{36}$ ou obtém-se o valor de concentração utilizando-se a Equação $27^{9}$ :

$\mathrm{TA}_{\mathrm{z}^{\prime}}=\mathrm{TA}_{\mathrm{z}} \frac{\mathrm{Z}^{\prime}}{\mathrm{Z}}$

onde: Z' = distância utilizada na amostragem (cm) e Z = distância utilizada na calibração $(\mathrm{cm})$.

$\mathrm{C}=\frac{\left(\frac{\mathrm{m} \times \mathrm{Z}^{\prime}}{\mathrm{t}}\right)}{\mathrm{TA} \times \mathrm{Z}}$

onde: $\mathrm{C}=$ concentração na amostra $\left(\mathrm{g} / \mathrm{cm}^{3}\right) ; \mathrm{m}=$ massa do analito na fibra $(\mathrm{g})$ e $\mathrm{t}=$ tempo de amostragem $(\mathrm{s})$.

\section{APLICAÇÕES DA AMOSTRAGEM DE COMPOSTOS VOLÁTEIS UTILIZANDO A TÉCNICA SPME}

A Tabela 3 apresenta um resumo de alguns trabalhos descritos na literatura sobre a utilização da técnica SPME para amostragem de diferentes compostos voláteis em matrizes variadas.

Nos estudos citados, nota-se que a técnica de SPME possibilitou a obtenção de menores tempos de amostragem e análise, com sensibilidade maior que os métodos de referência atualmente utili$\operatorname{zados}^{3,9,12,34}$. A principal interferência observada nos trabalhos que utilizavam fibras porosas foi a saturação e competição por sítios ativos da fase devido à presença de vários COV na matriz, como mostra o trabalho de amostragem de compostos voláteis de enxofre $^{44}$. Para uma amostragem e análise com boa exatidão, a umidade do local estudado deve ser considerada no processo de quantificação de COV, conforme discutido anteriormente na descrição do processo de absorção ${ }^{23,24,29}$.

\section{REFERÊNCIAS}

1. Keith, L. H.; Principles of Environmental Sampling, $2^{\text {nd }}$ ed., ACS: Washington, 1996.

2. Camel, V.; Caude, M.; J. Chromatogr., A 1995, 710, 3.
3. Koziel, J.; Jia, M.; Khaled, A.; Noah, J.; Pawliszyn, J.; Anal. Chim. 1999, 400, 153.

4. Arthur, C. L.; Pawliszyn, J.; Anal. Chem. 1990, 62, 2145.

5. Eisert, R.; Pawliszyn, J.; Crit. Rev. Anal. Chem. 1997, 27, 103.

6. Pawliszyn, J.; Applications of Solid Phase Microextraction, Royal Society Chemistry: Cambridge, 1999.

7. Alpendurada, M. F.; J. Chromatogr., A 2000, 889, 3.

8. Fox, D. L.; Anal. Chem. 1999, 71, 109.

9. Martos, P. A.; Pawliszyn, J.; Anal. Chem. 1999, 71, 1513.

10. Bartelt, J. R.; Zilkowski, B. W.; Anal. Chem. 1999, 71, 92.

11. Koziel, J.; Jia, M.; Pawliszyn, J.; Anal. Chem. 2000, 72, 5178.

12. Chai, M.; Pawliszyn, J.; Environ. Sci. Technol. 1995, 29, 693.

13. Müller, L.; Górecki, T.; Pawliszyn, J.; Fresenius Journal Anal. Chem. 1999, 364,610 .

14. Mani, V. Em Applications of Solid Phase Microextraction; Pawliszyn, J., ed.; Royal Society Chemistry: Cambridge, 1999, cap. 5.

15. Pawliszyn, J.; Solid Phase Microextraction - Theory and Practice; WileyVCH Inc.: New York, 1997.

16. Benfenati, E; Müller; Perani, L; Pierucci, P. Em ref. 14, cap. 25.

17. Górecki, T. Em ref. 14, cap. 7.

18. Pawliszyn, J.; J. Chromatogr. Sci. 2000, 38, 270.

19. Martos, P. A.; Saraullo, A.; Pawliszyn, J.; Anal. Chem. 1997, 69, 402.

20. Martos, P. A.; Pawliszyn, J.; Anal. Chem. 1997, 69, 206.

21. Sadtler; The Sadtler Capillary GC Standard Retention Index Library and Data Base, Sadtler Research Laboratories: Philadelphia, PA.

22. Bartelt, J. R.; Zilkowski, B.W.; Anal. Chem. 2000, 72, 3949.

23. Gorlo, D.; Wolska, L.; Zygmunt, B.; Namieśnik, J.; Talanta 1997, 44, 1543.

24. Namiesnik, J.; Gorlo, D.; Wolska, L.; Zygmunt, B.; Analusis 1998, 26, 170.

25. Namiesnik, J.; Zygmunt, B.; Jastrzębska, A.; J. Chromatogr., A 2000, 885, 405.

26. Chai, M.; Arthur, C. L.; Pawliszyn, J.; Belardi, R. P.; Pratt, K. F.; Analyst 1993, 118, 1501.

27. Grote, C.; Pawliszyn, J.; Anal. Chem. 1997, 69, 587.

28. Bolta, Š. V.; Zupannčičkralj, L.; Marsel, J.; Chromatographia 1998, 48, 95.

29. Gorlo, D.; Zygmunt, B.; Dudek, M.; Pilarczyk, M.; Namieśnik, J.; Fresenius J. Anal. Chem. 1999, 363, 696.

30. Martos, P. A. Em ref. 14, cap. 11.

31. Chai, M.; Tang, Y-Z.; Int. J. Environ. Anal. Chem. 1998, 72, 77.

32. Martos, P. A.; Pawliszyn, J.; Anal. Chem. 1998, 70, 2311.

33. Stashenko, E. E.; Puertas, M. A.; Slagar, W.; Delgado, W.; Martinez, R. S.; J. Chromatogr., A 2000, 886, 175.

34. Koziel, J. A.; Noah, J.; Pawliszyn, J.; Environ. Sci. Technol. 2001, 35, 1481.

35. Tuduri, l.; Desausiers, V.; Fanilo, J. L.; J. Chromatogr., A 2002, 963, 49.

36. Khaled, A; Pawlizyn, J.; J. Chromatogr., A 2000, 892, 455.

37. Tsai, S-W.; Chang, T-A.; J. Chromatogr., A 2002, 954, 198.

38. http://www.supelco.com, acessada em Fevereiro 2002.

39. Llompart, M.; Li, K.; Fingas, M.; J. Chromatogr. 1998, 824, 53.

40. Elke, K.; Jermann, E.; Begerow, J.; Dunemann, L.; J. Chromatogr. 1998, $826,191$.

41. Saba, A.; Raffaelli, S.; Pucci, S.; Salvadori, P.; Rapid Commun. Mass Spectrom. 1999, 13, 1899.

42. Parreira, F. V; Carvalho, C. R.; Cardeal, Z. L; Bull. Environ. Contam. Toxicol. 2003, 70, 957.

43. Li, K.; Santilli, A.; Goldthorp, M.; Whiticar, S.; Lambert, P.; Fingas, M.; J. Hazard. Mater. 2001, 83, 83.

44. Nielsen, A.; Jonsson, S.; J. Chromatogr. 2002, 963, 57.

45. Chien, Y-C.; Uang, S-N.; Kuo, C-T.; Shih, T-S.; Jen, J-F.; Anal. Chim. 2000, $419,73$. 